\title{
Building bases for analytical fits of four-Ioop QED master integrals
}

\section{Stefano Laporta*}

Dipartimento di Fisica e Astronomia, Università di Padova, Via Marzolo 8, I-35131 Padova, Italy

Istituto Nazionale Fisica Nucleare, Sezione di Padova, Via Marzolo 8, I-35131 Padova, Italy E-mail: stefano.laporta@pd.infn.it

In this paper I will briefly describe how to find some elements of the basis necessary for the PSLQ fits of master integrals that appear in the calculation of four-loop contributions to the electron $g$ - 2 and the renormalization constants $Z_{2}$ and $Z_{m}$ in QED. In particular I consider master integrals containing polylogarithms of the sixth root of the unity and elliptical integrals. A new highprecision numerical determination of $Z_{2}$ at four loops will be also shown.

Loops and Legs in Quantum Field Theory (LL2018)

29 April 2018 - 04 May 2018

St. Goar, Germany

${ }^{*}$ Speaker. 
There are 891 four-loop Feynman diagrams which contribute [1] to the 4-loop $g$-2 in QED. Some diagrams are shown in Fig. 1. The contribution of each diagram can be reduced to linear combinations of 334 master integrals with polynomials in the number of dimensions $D$ as

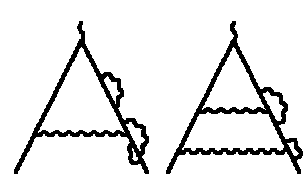

(1)

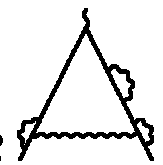

(3)

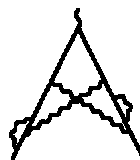

(4)

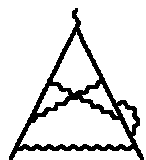

(5)

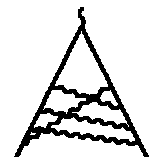

(6)

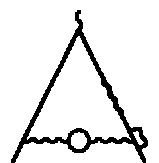

(7)

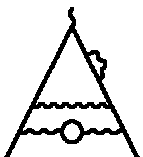

(8)

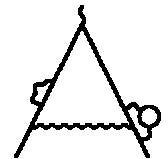

(9)

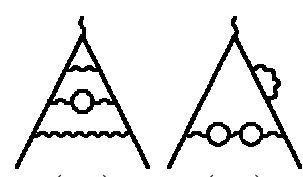

(11)

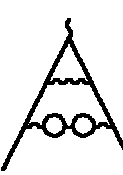

(12)

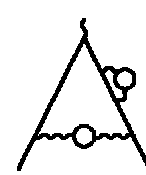

(13)

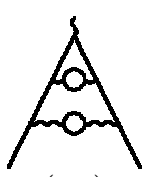

(14)

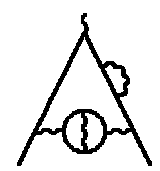

(15)

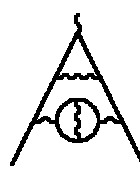

(16)

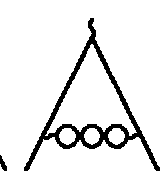

(17)

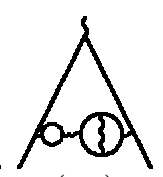

(18)

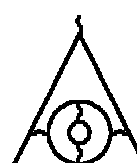

(19)

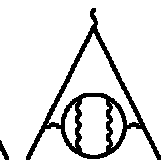

(20)

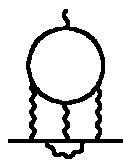

(21)

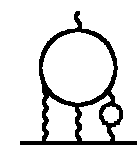

(22)

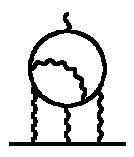

(23)

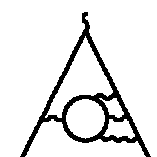

(24)

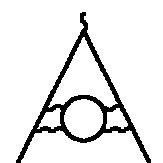

(25)

Figure 1: Typical 4-loop vertex diagrams.

coefficients by using integration by parts identities $[2,3,4,5]$. The needed coefficients of the $\varepsilon$-expansions of the master integrals were calculated with at least 1100 digits of precision in Ref. [1]. Some of them were calculated up to 9600 digits. These high precision results were needed to fit analytical expressions to the numerical values by means of the PSLQ algorithm [6, 7].

The algorithm PSLQ (Ref. [6]) can be seen as a multidimensional extension of the well-known Euclid algorithm for the calculation of the GCD of two integers. It finds linear relations with integer coefficients between high-precision numerical constants or, alternatively, it gives limits on the size of the coefficients. Usually it needs values with very high precision.

First of all, let us recall the structure of the analytical fit to the numerical value of the 4-loop contribution to the electron $g$-2 in QED obtained in Ref. [1]:

$$
A_{1}^{(8)}=T+\sqrt{3} V_{a}+V_{b}+W_{b}+\sqrt{3} E_{a}+E_{b}+U ;
$$

The various pieces contain constants belonging to different families: $T$ contains values of Harmonic Polylogarithms (HPL) [8] $H_{\{i\}}(x)$ with argument $x=\frac{1}{2}$ or $x=1 . V_{a}$ and $V_{b}$ contain values of HPLs at $e^{\frac{i \pi}{3}}$ or $e^{\frac{2 i \pi}{3}} . W_{a}$ contains values of HPLs at $e^{\frac{i \pi}{2}} . E_{a}$ and $E_{b}$ contain elliptical integrals. We will show some example for each family.

\section{Family 1: harmonic polylogarithms of $\frac{1}{2}$ or 1}

The total number of elements and some elements are listed in table 1. The number of elements with weight $w$ is the Fibonacci number $F_{w+1}$. The total number of elements up to weight $w$ if $F_{2 w+3}-1$. The Fibonacci numbers are the solutions of the recurrence relation $F_{n+1}=F_{n}+F_{n-1}$ 


\begin{tabular}{rrl}
\hline weight & number & constants \\
\hline 0 & 1 & 1 \\
1 & 1 & $\ln 2$ \\
2 & 2 & $\zeta(2), \ln ^{2} 2$ \\
3 & 3 & $\zeta(3), \zeta(2) \ln 2, \ln ^{3} 2$ \\
4 & 5 & $\zeta(4), \zeta(3) \ln 2, \zeta(2) \ln ^{2} 2, \ln ^{4} 2, a_{4}$ \\
5 & 8 & $\zeta(5), \zeta(4) \ln 2, \zeta(3) \ln ^{2} 2, \zeta(2) \ln ^{3} 2, \zeta(3) \zeta(2), a_{4} \ln 2, \ln ^{5} 2, a_{5}$ \\
6 & 13 & $\zeta(6), \zeta(3)^{2}, \ldots, a_{5} \ln 2, \ln ^{6} 2, a_{6}, b_{6}$ \\
7 & 21 & $\zeta(7), \zeta(4) \zeta(3), \ldots, b_{6} \ln 2, \ln ^{7} 2, a_{7}, b_{7}, d_{7}$ \\
$w$ & $F_{w+1}$ & \\
\hline
\end{tabular}

Table 1: Number and elements of the family of harmonic polylogarithms of argument $\frac{1}{2}$ and $1 . \zeta(n)=$ $\operatorname{Li}_{n}(1), a_{n}=\mathrm{Li}_{n}(1 / 2), b_{6}=H_{0,0,0,0,1,1}(1 / 2), b_{7}=H_{0,0,0,0,0,1,1}(1 / 2)$ and $d_{7}=H_{0,0,0,0,1,-1,-1}(1)$.

with the initial condition $F_{0}=F_{1}=1$.

As an example of a typical fit, we consider the 7-lines master integral $(D=4-2 \varepsilon)$

$$
\left[\pi^{-\frac{D}{2}} \Gamma(1+\varepsilon)\right]^{4} \bigcirc=G_{1} \varepsilon^{-4}+G_{2} \varepsilon^{-3}+G_{3} \varepsilon^{-2}+G_{4} \varepsilon^{-1}+G_{5}+G_{6} \varepsilon+G_{7} \varepsilon^{2}+\ldots
$$

The first seven constants $G_{i}$ were calculated numerically with 1200 digits and fitted with PSLQ; the results are

$$
\begin{aligned}
& G_{1}=-\frac{1}{8}, G_{2}=-\frac{49}{48}, G_{3}=-\frac{449}{96}-\frac{1}{6} \pi^{2}-\frac{1}{2} \zeta(3), G_{4}=-\frac{2429}{192}-\frac{7}{4} \pi^{2}-\frac{11}{2} \zeta(3)-\frac{1}{120} \pi^{4}, \\
& G_{5}=\frac{2687}{384}-\frac{277}{24} \pi^{2}-\frac{125}{3} \zeta(3)+\frac{1}{24} \pi^{4}+\frac{3}{2} \zeta(5)+\frac{2}{3} \pi^{2} \zeta(3), \\
& G_{6}=\frac{95689}{256}-\frac{2377}{48} \pi^{2}-\frac{2999}{12} \zeta(3)-\frac{46}{45} \pi^{4}+\frac{149}{2} \zeta(5)+\frac{15}{2} \zeta(3)^{2}-\frac{58}{3} \pi^{2} \zeta(3)-\frac{29}{270} \pi^{6}, \\
& G_{7}=\frac{1671597}{512}-\frac{4381}{96} \pi^{2}-\frac{22193}{24} \zeta(3)-144 \pi^{2} \ln 2-\frac{3617}{240} \pi^{4}-\frac{71}{2} \zeta(5)-\frac{393}{2} \pi^{2} \zeta(3)-\frac{869}{162} \pi^{6} \\
& +24 \pi^{2}\left(\ln ^{4} 2-\pi^{2} \ln ^{2} 2+24 a_{4}\right)-\frac{803}{2} \zeta(3)^{2}+504 \pi^{2} \zeta(3) \ln 2-\frac{1735}{4} \zeta(7)+\frac{799}{6} \pi^{2} \zeta(5)-\frac{661}{180} \pi^{4} \zeta(3) .
\end{aligned}
$$

For example, $G_{7}$ contains constants up to weight seven, so that the needed basis contains 54 terms; this is the output of the PSLQM program [9]:

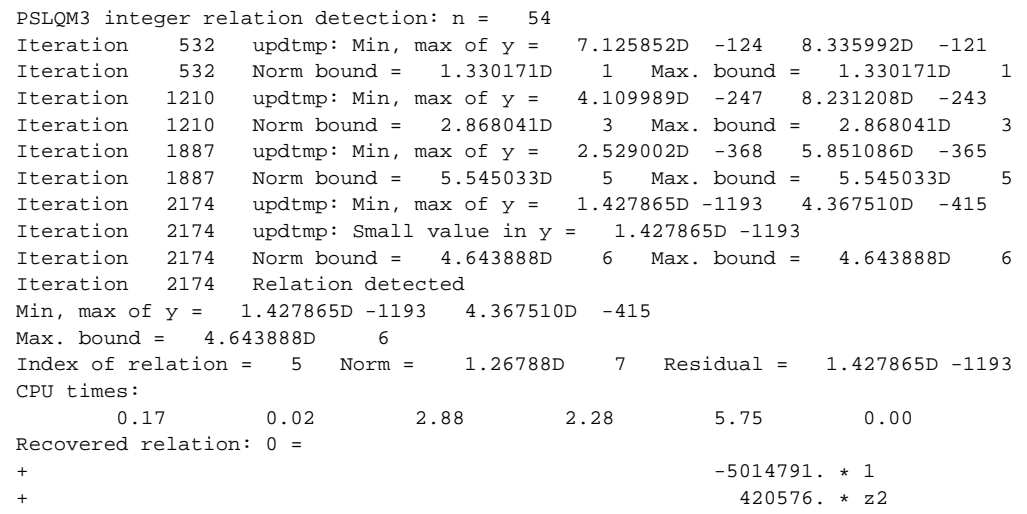



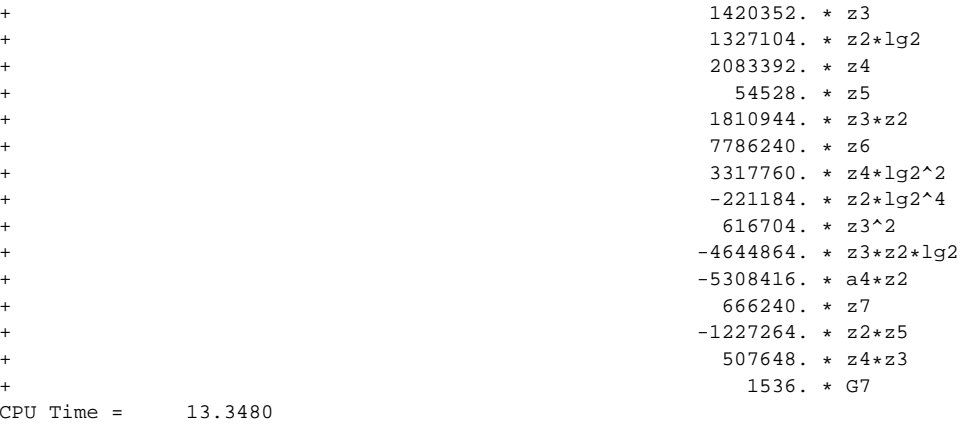

Note that only 415 digits are needed to find this fit, the remaining digits are a "safety factor".

The complete analytic expression of $T$ of Eq. (1) is [1]:

$$
\begin{aligned}
T= & \frac{1243127611}{130636800}+\frac{30180451}{25920} \zeta(2)-\frac{255842141}{2721600} \zeta(3)-\frac{8873}{3} \zeta(2) \ln 2+\frac{6768227}{2160} \zeta(4)+\frac{19063}{360} \zeta(2) \ln ^{2} 2 \\
& +\frac{12097}{90}\left(a_{4}+\frac{1}{24} \ln ^{4} 2\right)-\frac{2862857}{6480} \zeta(5)-\frac{12720907}{64800} \zeta(3) \zeta(2)-\frac{221581}{2160} \zeta(4) \ln 2 \\
& +\frac{9656}{27}\left(a_{5}+\frac{1}{12} \zeta(2) \ln ^{3} 2-\frac{1}{120} \ln ^{5} 2\right)+\frac{191490607}{46656} \zeta(6)+\frac{10358551}{43200} \zeta^{2}(3)-\frac{40136}{27} a_{6}+\frac{26404}{27} b_{6} \\
& -\frac{700706}{675} a_{4} \zeta(2)-\frac{26404}{27} a_{5} \ln 2+\frac{26404}{27} \zeta(5) \ln 2-\frac{63749}{50} \zeta(3) \zeta(2) \ln 2-\frac{40723}{135} \zeta(4) \ln ^{2} 2+\frac{13202}{81} \zeta(3) \ln ^{3} 2 \\
& -\frac{253201}{2700} \zeta(2) \ln ^{4} 2+\frac{7657}{1620} \ln ^{6} 2+\frac{2895304273}{435456} \zeta(7)+\frac{670276309}{193536} \zeta(4) \zeta(3)+\frac{85933}{63} a_{4} \zeta(3)-\frac{142793}{18} a_{5} \zeta(2) \\
& +\frac{7121162687}{967680} \zeta(5) \zeta(2)-\frac{195848}{21} a_{7}+\frac{195848}{63} b_{7}-\frac{116506}{189} d_{7}-\frac{4136495}{384} \zeta(6) \ln 2-\frac{1053568}{189} a_{6} \ln 2 \\
& +\frac{233012}{189} b_{6} \ln 2+\frac{407771}{432} \zeta^{2}(3) \ln 2-\frac{8937}{2} a_{4} \zeta(2) \ln 2+\frac{833683}{3024} \zeta(5) \ln ^{2} 2-\frac{3995099}{6048} \zeta(3) \zeta(2) \ln ^{2} 2 \\
& -\frac{233012}{189} a_{5} \ln ^{2} 2+\frac{1705273}{1512} \zeta(4) \ln ^{3} 2+\frac{602303}{4536} \zeta(3) \ln ^{4} 2-\frac{1650461}{11340} \zeta(2) \ln ^{5} 2+\frac{52177}{15876} \ln ^{7} 2 .
\end{aligned}
$$

It contains 46 terms of the 54 terms of general basis of HPLs of argument $\frac{1}{2}$ and 1 up to weight 7 .

\section{Family 2: values of harmonic polylogarithms of argument $e^{\frac{i \pi}{3}}$ and $e^{\frac{2 i \pi}{3}}$}

The number and some elements are listed in table 2. The number of the elements of the subsets with weight $w$ composed only by real or imaginary parts of (products of) HPLs are $\left(F_{2 w+2}+\right.$ $\left.F_{w+1}\right) / 2$ and $\left(F_{2 w+2}-F_{w+1}\right) / 2$, respectively. The total numbers of elements up to weight $w$ is $\left(F_{2 w+3}+F_{w+3}\right) / 2-1$ and $\left(F_{2 w+3}-F_{w+3}\right) / 2$, for real and imaginary parts, respectively.

Let us consider now the two master integrals of Fig. 2; by using HPLs of argument $\frac{1}{2}$ and 1 as basis, PSLQ fits only the divergent terms:

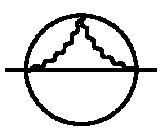

(a)

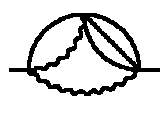

(b)

Figure 2: The simplest master integrals which contain HPLs of argument $e^{\frac{i \pi}{3}}$. 


\begin{tabular}{rrl}
\hline weight & \#re+im & constants \\
\hline 0 & $1+0$ & 1 \\
1 & $2+1$ & $\ln 2, \ln 3, \pi \sqrt{3}$ \\
2 & $5+3$ & $\zeta(2), \ln 22, \ln 2 \ln 3, \ln ^{2} 3, \operatorname{Re}\left(H_{1,-1}\left(e^{i \pi / 3}\right)\right), \pi \ln 2 \sqrt{3}, \pi \ln 3 \sqrt{3}, \mathrm{Cl}_{2}\left(\frac{\pi}{3}\right) \sqrt{3}$ \\
3 & $12+9$ & $\zeta(3), \pi \mathrm{Cl}_{2}\left(\frac{\pi}{3}\right), \operatorname{Re}\left(H_{1,1,-1}\left(e^{i \pi / 3}\right)\right), \operatorname{Im}\left(H_{0,1,1}\left(e^{2 i \pi / 3}\right)\right) \sqrt{3}, \operatorname{Im}\left(H_{0,1,-1}\left(e^{i \pi / 3}\right)\right) \sqrt{3}, \ldots$ \\
4 & $30+25$ & $\zeta(4), \operatorname{Re}\left(H_{0,0,1,1}\left(e^{2 i \pi / 3}\right)\right), \operatorname{Im}\left(H_{0,1,1,1}\left(e^{2 i \pi / 3}\right)\right) \sqrt{3}, \operatorname{Im}\left(H_{0,1,1,-1}\left(e^{i \pi / 3}\right)\right) \sqrt{3}, \ldots$ \\
5 & $76+68$ & $\zeta(5), \operatorname{Re}\left(H_{0,0,1,1,1}\left(e^{2 i \pi / 3}\right)\right), \operatorname{Im}\left(H_{0,0,0,1,1}\left(e^{i \pi / 3}\right)\right) \sqrt{3}, \ldots$ \\
6 & $195+182$ & $\zeta(6), \operatorname{Re}\left(H_{0,0,1,1,0,1}\left(e^{i \pi / 3}\right)\right), \operatorname{Im}\left(H_{0,0,0,1,1,1}\left(e^{2 i \pi / 3}\right)\right) \sqrt{3}, \ldots$ \\
7 & $504+483$ & $\zeta(7), \operatorname{Re}\left(H_{0,0,0,0,1,1,1}\left(e^{2 i \pi / 3}\right)\right), \operatorname{Im}\left(H_{0,0,0,0,0,1,1}\left(e^{i \pi / 3}\right)\right) \sqrt{3}, \ldots$ \\
$w$ & $F_{2 w+2}$ & \\
\hline
\end{tabular}

Table 2: Number and elements of the family of harmonic polylogarithms of argument $e^{\frac{i \pi}{3}}$ and $e^{\frac{2 i \pi}{3}}$

$$
\begin{aligned}
& I(a)=\frac{7}{12 \varepsilon^{4}}+\frac{10}{3 \varepsilon^{3}}+\frac{121}{12 \varepsilon^{2}}+\left(\frac{1541}{72}+\frac{7}{6} \zeta(3)\right) \varepsilon^{-1}+X_{a}^{(0)}+O(\varepsilon), \\
& I(b)=\frac{5}{8 \varepsilon^{4}}+\frac{59}{16 \varepsilon^{3}}+\left(\frac{1099}{36}+3 \zeta(2)\right) \varepsilon^{-2}+\left(\frac{3781}{192}+\frac{33}{2} \zeta(2)+6 \zeta(3)\right) \varepsilon^{-1}+X_{b}^{(0)}+O(\varepsilon) .
\end{aligned}
$$

$X_{a}^{(0)}$ and $X_{b}^{(0)}$ contain other constants. We observe that, by closing the external line of the diagrams (a) or (b) with a massive line, we obtain the same vacuum diagram, so we expect that $X_{a}^{(0)}$ and $X_{b}^{(0)}$ contain the same constants.

The first step in identifying the family of constants is to write (part of) the constants terms as integral; we consider the hyperspherical representation [10] in two space-time dimensions

$$
\pi^{-2} \overbrace{m_{3}}^{m_{1}} p=\int_{0}^{\infty} \frac{d l}{l+m_{1}^{2}} \int_{0}^{\infty} \frac{d r}{R\left(l, r,-m_{2}^{2}\right) R\left(r, p^{2},-m_{3}^{2}\right)}
$$

where $R(x, y, z)=\sqrt{(x-y-z)^{2}-4 y z}$ is the well-known Källen function. Note that Eq. (2.3) is always valid for spacelike momenta $p$; the analytic continuation to timelike moments may need a deformation of the contours of the radial integration over $l$ and $r$, not needed for the subsequent discussion. Setting $m_{i}=1, p^{2}=-1$,

$$
\begin{aligned}
& \pi^{-2}=\int_{0}^{\infty} d l \frac{1}{l+1} \int_{0}^{\infty} \frac{d r}{R(l, r,-1) R(r,-1,-1)}, \\
& \pi^{-2} \rightarrow \int_{0}^{\infty} l d l\left(\frac{\ln (l+1)}{l}\right)^{2} \int_{0}^{\infty} \frac{d r}{R(l, r,-1) R(r,-1,-1)} .
\end{aligned}
$$

If we define the ad hoc family of integrals

$$
I\left(n_{1}, n_{2}\right)=\int_{0}^{\infty} \frac{d l}{l} \ln ^{n_{1}} l \ln ^{n_{2}}(l+1) \ldots \int_{0}^{\infty} \frac{d r}{R(l, r,-1) R(r,-1,-1)}
$$


PSLQ gives

$$
X_{a}^{(0)}=\frac{42155}{432}-\frac{380}{3} \zeta(2)+\frac{14}{3} \zeta(3)+\frac{3}{2} \zeta(4)-\frac{3}{2} I(0,2) .
$$

Now $I(0,2)$ can be integrated analytically, and one finds HPLs of argument $e^{i \pi / 3}$, in this case the Clausen's function. Using the family 2 as basis, one finds the fits

$$
\begin{aligned}
& X_{a}^{(0)}=\frac{42155}{432}-\frac{380}{3} \zeta(2)+\frac{14}{3} \zeta(3)+\frac{3}{2} \zeta(4)+\sqrt{3}\left(6 \mathrm{Cl}_{4}\left(\frac{\pi}{3}\right)-10 \zeta(2) \mathrm{Cl}_{2}\left(\frac{\pi}{3}\right)\right), \\
& X_{b}^{(0)}=\frac{25033}{1152}-\frac{47}{4} \zeta(2)+\frac{69}{2} \zeta(3)+\frac{411}{8} \zeta(4)-\sqrt{3}\left(9 \mathrm{Cl}_{4}\left(\frac{\pi}{3}\right)+9 \zeta(2) \mathrm{Cl}_{2}\left(\frac{\pi}{3}\right)\right) .
\end{aligned}
$$

Out of curiosity, the fit of the coefficients of the $\varepsilon, \varepsilon^{2}, \varepsilon^{3}$ terms of the $\varepsilon$-expansion of $I(a)$ turn out to contain 18, 61 and 199 terms, respectively. The complete analytic expression of $V_{a}$ and $V_{b}$ of Eq. (1) are [1]:

$$
\begin{aligned}
& V_{a}=-\frac{14101}{480} \mathrm{Cl}_{4}\left(\frac{\pi}{3}\right)-\frac{169703}{1440} \zeta(2) \mathrm{Cl}_{2}\left(\frac{\pi}{3}\right)+\frac{494}{27} \operatorname{Im} H_{0,0,0,1,-1,-1}\left(e^{i \frac{\pi}{3}}\right)+\frac{494}{27} \operatorname{Im} H_{0,0,0,1,-1,1}\left(e^{i \frac{2 \pi}{3}}\right) \\
& +\frac{494}{27} \operatorname{Im} H_{0,0,0,1,1,-1}\left(e^{i \frac{2 \pi}{3}}\right)+19 \operatorname{Im} H_{0,0,1,0,1,1}\left(e^{i \frac{2 \pi}{3}}\right)+\frac{437}{12} \operatorname{Im} H_{0,0,0,1,1,1}\left(e^{i \frac{2 \pi}{3}}\right)+\frac{29812}{297} \mathrm{Cl}_{6}\left(\frac{\pi}{3}\right) \\
& +\frac{4940}{81} a_{4} \mathrm{Cl}_{2}\left(\frac{\pi}{3}\right)-\frac{520847}{69984} \zeta(5) \pi-\frac{129251}{81} \zeta(4) \mathrm{Cl}_{2}\left(\frac{\pi}{3}\right)-\frac{892}{15} \operatorname{Im} H_{0,1,1,-1}\left(e^{i \frac{2 \pi}{3}}\right) \zeta(2) \\
& -\frac{1784}{45} \operatorname{Im} H_{0,1,1,-1}\left(e^{i \frac{\pi}{3}}\right) \zeta(2)+\frac{1729}{54} \zeta(3) \operatorname{Im} H_{0,1,-1}\left(e^{i \frac{\pi}{3}}\right)+\frac{1729}{36} \zeta(3) \operatorname{Im} H_{0,1,1}\left(e^{i \frac{2 \pi}{3}}\right) \\
& +\frac{837190}{729} \mathrm{Cl}_{4}\left(\frac{\pi}{3}\right) \zeta(2)+\frac{25937}{4860} \zeta(3) \zeta(2) \pi-\frac{223}{243} \zeta(4) \pi \ln 2+\frac{892}{9} \operatorname{Im} H_{0,1,-1}\left(e^{i \frac{\pi}{3}}\right) \zeta(2) \ln 2 \\
& +\frac{446}{3} \operatorname{Im} H_{0,1,1}\left(e^{i \frac{2 \pi}{3}}\right) \zeta(2) \ln 2-\frac{7925}{81} \mathrm{Cl}_{2}\left(\frac{\pi}{3}\right) \zeta(2) \ln ^{2} 2+\frac{1235}{486} \mathrm{Cl}_{2}\left(\frac{\pi}{3}\right) \ln ^{4} 2, \\
& V_{b}=\frac{13487}{60} \operatorname{Re} H_{0,0,0,1,0,1}\left(e^{i \frac{\pi}{3}}\right)+\frac{13487}{60} \mathrm{Cl}_{4}\left(\frac{\pi}{3}\right) \mathrm{Cl}_{2}\left(\frac{\pi}{3}\right)+\frac{136781}{360} \mathrm{Cl}_{2}^{2}\left(\frac{\pi}{3}\right) \zeta(2)+\frac{651}{4} \operatorname{Re} H_{0,0,0,1,0,1,-1}\left(e^{i \frac{\pi}{3}}\right) \\
& +651 \operatorname{Re} H_{0,0,0,0,1,1,-1}\left(e^{i \frac{\pi}{3}}\right)-\frac{17577}{32} \operatorname{Re} H_{0,0,1,0,0,1,1}\left(e^{i \frac{2 \pi}{3}}\right)-\frac{87885}{64} \operatorname{Re} H_{0,0,0,1,0,1,1}\left(e^{i \frac{2 \pi}{3}}\right) \\
& -\frac{17577}{8} \operatorname{Re}_{0,0,0,0,1,1,1}\left(e^{i \frac{2 \pi}{3}}\right)+\frac{651}{4} \mathrm{Cl}_{4}\left(\frac{\pi}{3}\right) \operatorname{Im} H_{0,1,-1}\left(e^{i \frac{\pi}{3}}\right)+\frac{1953}{8} \mathrm{Cl}_{4}\left(\frac{\pi}{3}\right) \operatorname{Im} H_{0,1,1}\left(e^{i \frac{2 \pi}{3}}\right) \\
& +\frac{31465}{176} \mathrm{Cl}_{6}\left(\frac{\pi}{3}\right) \pi+\frac{211}{4} \operatorname{Re}_{0,1,0,1,-1}\left(e^{i \frac{\pi}{3}}\right) \zeta(2)+\frac{211}{2} \operatorname{Re}_{0,0,1,1,-1}\left(e^{i \frac{\pi}{3}}\right) \zeta(2) \\
& +\frac{1899}{16} \operatorname{Re} H_{0,1,0,1,1}\left(e^{i \frac{2 \pi}{3}}\right) \zeta(2)+\frac{1899}{8} \operatorname{Re} H_{0,0,1,1,1}\left(e^{i \frac{2 \pi}{3}}\right) \zeta(2)+\frac{211}{4} \operatorname{Im}_{0,1,-1}\left(e^{i \frac{\pi}{3}}\right) \mathrm{Cl}_{2}\left(\frac{\pi}{3}\right) \zeta(2) \\
& +\frac{633}{8} \operatorname{Im} H_{0,1,1}\left(e^{i \frac{2 \pi}{3}}\right) \mathrm{Cl}_{2}\left(\frac{\pi}{3}\right) \zeta(2) \text {. }
\end{aligned}
$$

$V_{a}$ and $V_{b}$, composed by imaginary and real parts of HPLs, contain 23 terms and 17 terms, respectively, to be compared with the length of the general basis up to weight seven which is 671 and 825 terms, respectively. Therefore only a small part of the general basis is really needed to fit $V_{a}$ and $V_{b}$.

\section{Family 3: values of harmonic polylogarithms of argument $e^{\frac{i \pi}{2}}$.}

The number and some elements are listed in table 3. The number of the elements of the subsets with weight $w$ composed only by real or imaginary parts of (products of) HPLs is $2^{w}$. The analytical expression of $W_{b}$ is [1]:

$$
W_{b}=\zeta(2)\left(-\frac{28276}{25} \mathrm{Cl}_{2}\left(\frac{\pi}{2}\right)^{2}+104\left(4 \operatorname{Re} H_{0,1,0,1,1}(i)+4 \operatorname{Im} H_{0,1,1}(i) \mathrm{Cl}_{2}\left(\frac{\pi}{2}\right)-2 \mathrm{Cl}_{4}\left(\frac{\pi}{2}\right) \pi+\mathrm{Cl}_{2}^{2}\left(\frac{\pi}{2}\right) \ln 2\right)\right) ;
$$




\begin{tabular}{rrl}
\hline weight & \#re+im & constants \\
\hline 0 & $1+0$ & 1 \\
1 & $1+1$ & $\ln 2, \pi$ \\
2 & $2+2$ & $\zeta(2), \ln ^{2} 2, \pi \ln 2, \beta_{2}$ \\
3 & $4+4$ & $\zeta(3), \pi \beta_{2}, \operatorname{Im}\left(H_{0,1,1}\left(e^{i \pi / 2}\right)\right), \ldots$ \\
4 & $8+8$ & $\zeta(4), \beta_{2}^{2}, \beta_{4}, \operatorname{Im}\left(H_{0,1,1,1}\left(e^{i \pi / 2}\right)\right), \ldots$ \\
5 & $16+16$ & $\zeta(5), \operatorname{Re}\left(H_{0,1,0,1,1}\left(e^{i \pi / 2}\right)\right), \operatorname{Im}\left(H_{0,0,0,1,1}\left(e^{i \pi / 2}\right)\right), \ldots$ \\
6 & $32+32$ & $\zeta(6), \operatorname{Re}\left(H_{0,0,0,1,0,1}\left(e^{i \pi / 2}\right)\right), \operatorname{Im}\left(H_{0,0,0,1,1,1}\left(e^{i \pi / 2}\right)\right), \ldots$ \\
7 & $64+64$ & $\zeta(7), \operatorname{Re}\left(H_{0,0,0,1,0,1,1}\left(e^{i \pi / 2}\right)\right), \operatorname{Im}\left(H_{0,0,0,0,0,1,1}\left(e^{i \pi / 2}\right)\right), \ldots$ \\
$w$ & $2^{w}$ & \\
\hline
\end{tabular}

Table 3: Number and elements of the family of harmonic polylogarithms of argument $e^{\frac{i \pi}{2}} ; \beta_{2}$ is the Catalan's constant

it contains only 5 terms, to be compared to the general basis which contains 256 terms. Therefore only a very small part of the general basis is needed to fit $W_{b}$.

\section{Elliptic master integrals}

The master integrals containing a massive 5-body cut require a new class of elliptic constants. A fit to the constant term of the $\varepsilon$-expansion of the simplest master integral of this class was found in Ref. [11]:

$$
\left[\pi^{-\frac{D}{2}} \Gamma(1+\varepsilon)\right]^{4} \bigcirc=-\frac{5}{2 \varepsilon^{4}}-\frac{45}{4 \varepsilon^{3}}-\frac{4255}{144 \varepsilon^{2}}-\frac{106147}{1728 \varepsilon}+\frac{\pi \sqrt{3}}{240}\left(297 B_{3}-1477 C_{3}\right)-\frac{2320981}{20736}+O(\varepsilon)
$$

The constants $B_{3}$ and $C_{3}$ are defined as

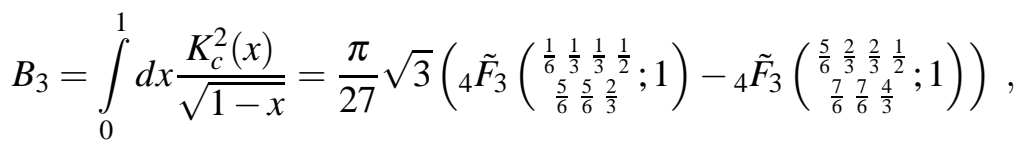

$$
\begin{aligned}
& C_{3}=\int_{0}^{1} d x \frac{E_{c}^{2}(x)}{\sqrt{1-x}}=\frac{\pi}{27} \sqrt{3}\left({ }_{4} \tilde{F}_{3}\left(\begin{array}{c}
\frac{1}{6} \frac{1}{3} \frac{4}{3}-\frac{1}{2} \\
-\frac{1}{6} \frac{5}{6} \frac{5}{3}
\end{array} 1\right)-{ }_{4} \tilde{F}_{3}\left(\begin{array}{c}
-\frac{7}{6}-\frac{1}{3} \frac{2}{3}-\frac{1}{2} \\
-\frac{5}{6} \frac{1}{6} \frac{1}{3}
\end{array} ; 1\right)\right), \\
& { }_{4} \tilde{F}_{3}\left(\begin{array}{c}
a_{1} a_{2} a_{3} a_{4} \\
b_{1} b_{2} b_{3}
\end{array} ; x\right)=\frac{\Gamma\left(a_{1}\right) \Gamma\left(a_{2}\right) \Gamma\left(a_{3}\right) \Gamma\left(a_{4}\right)}{\Gamma\left(b_{1}\right) \Gamma\left(b_{2}\right) \Gamma\left(b_{3}\right)}{ }_{4} F_{3}\left(\begin{array}{c}
a_{1} a_{2} a_{3} a_{4} \\
b_{1} b_{2} b_{3}
\end{array} ; x\right) \\
& K_{c}(x)=\frac{2 \pi}{\sqrt{27}} 2 F_{1}\left(\begin{array}{c}
\frac{1}{3} \frac{2}{3} \\
1
\end{array} ; x\right), \quad E_{c}(x)=\frac{2 \pi}{\sqrt{27}} 2 F_{1}\left(\begin{array}{c}
\left.\frac{1}{3}-\frac{1}{3} ; x\right) \\
1
\end{array}\right.
\end{aligned}
$$

the representation (4.3) of $C_{3}$ in terms of the ${ }_{4} F_{3}$ hypergeometric function was found in Ref. [12]. Concerning the subsequent terms of the $\varepsilon$-expansion of this and other elliptic master integrals, the basis has to be found empirically. We close the external line of four-loop massive sunrise and we 
recognize that the vacuum integral contains two identical two-loop sunrise diagrams,

$$
\begin{aligned}
\pi^{-8} \rightarrow \pi^{-8} & =\int_{0}^{\infty} d l\left(\int_{0}^{\infty} \frac{d r}{R(l, r,-1) R(r,-1,-1)}\right)^{2} \\
& \rightarrow \int_{9}^{\infty} d s\left(\int_{9}^{\infty} \frac{d b}{R(s, b, 1) R(s, 1,1)}\right)^{2}=\int_{9}^{\infty} d s\left(D_{1}(s)\right)^{2}
\end{aligned}
$$

where

$$
D_{m}(s)=\frac{2}{\sqrt{(\sqrt{s}+3)(\sqrt{s}-1)^{3}}} K\left(m-1-(2 m-3) \frac{(\sqrt{s}-3)(\sqrt{s}+1)^{3}}{(\sqrt{s}+3)(\sqrt{s}-1)^{3}}\right), m=1,2 .
$$

$K(x)$ is the complete elliptic integral of the first kind; $D_{1}(s)$ is the discontinuity of the 2-loop sunrise diagram with equal masses in $D=2$ dimensions (see Ref. [13]). We define the family of integrals

$$
f_{m}(i, j, k)=\int_{1}^{9} d s D_{1}(s) \operatorname{Re}\left(\sqrt{3^{m-1}} D_{m}(s)\right)\left(s-\frac{9}{5}\right) \ln ^{i}(9-s) \ln ^{j}(s-1) \ln ^{k}(s), m=1,2,
$$

where $(s-9 / 5)$ is an ad-hoc factor which simplifies the basis. The elements of this family suffice to fit the combinations of the coefficients of the $\varepsilon$-expansions of the elliptic master integrals occurring in the contributions of diagrams. But to fit each coefficient one needs to enlarge the basis including also factors like $\ln (s+3), \mathrm{Li}_{2}(s / 9), \mathrm{Li}_{2}((s-1) / 8, \ldots$, and/or integrating on different intervals $([0,1],[9, \infty])$. For example the fit of the $\varepsilon^{3}$ coefficient of the integral 4.1 requires 187 elliptic elements, most of them outside the basis $\left\{f_{m}(i, j, k)\right\}$. Here we show the analytical expressions of $E_{a}$ and $E_{b}$ from Eq. (1):

$$
\begin{aligned}
E_{a}= & \pi\left(-\frac{28458503}{691200} B_{3}+\frac{250077961}{18662400} C_{3}\right)+\frac{483913}{77760} \pi f_{2}(0,0,1)+\pi\left(\frac{4715}{1944} \ln 2 f_{2}(0,0,1)+\frac{270433}{10935} f_{2}(0,2,0)\right. \\
& \left.-\frac{188147}{4860} f_{2}(0,1,1)+\frac{188147}{12960} f_{2}(0,0,2)\right)+\pi\left(\frac{826595}{248832} \zeta(2) f_{2}(0,0,1)-\frac{5525}{432} \ln 2 f_{2}(0,0,2)\right. \\
& +\frac{5525}{162} \ln 2 f_{2}(0,1,1)-\frac{5525}{243} \ln 2 f_{2}(0,2,0)+\frac{526015}{248832} f_{2}(0,0,3)-\frac{4675}{768} f_{2}(0,1,2)+\frac{1805965}{248832} f_{2}(0,2,1) \\
& \left.-\frac{3710675}{1119744} f_{2}(0,3,0)-\frac{75145}{124416} f_{2}(1,0,2)-\frac{213635}{124416} f_{2}(1,1,1)+\frac{168455}{62208} f_{2}(1,2,0)+\frac{69245}{124416} f_{2}(2,1,0)\right), \\
E_{b}= & \zeta(2)\left(\frac{2541575}{82944} f_{1}(0,0,2)-\frac{556445}{6912} f_{1}(0,1,1)+\frac{54515}{972} f_{1}(0,2,0)-\frac{75145}{20736} f_{1}(1,0,1)\right)-\frac{4715}{1458} \zeta(2) f_{1}(0,0,1) .
\end{aligned}
$$

We do not consider here $U$, which contains six coefficients of expansions of master integrals, not fitted analytically so far (see Ref. [1]).

\section{Other 4-loop quantities: $Z_{2}$ and $Z_{m}$ in QED}

One can wonder whether the basis so far obtained is sufficient to fit analytically other 4-loop QED quantities. We found that the 4-loop contributions to QED mass renormalization constant 
$Z_{m}^{O S}$ and the wave function renormalization constant $Z_{2}^{O S}$ can be fitted with the same basis used for the $g$-2. The number of terms of the analytical expression of $F_{2}(0)^{(4)}, Z_{2}^{(4)}(0)$ and $Z_{m}^{(4)}(0)$ are 121 , 118 and 73, respectively. Let us see the numerical value of $Z_{2}^{(4)}$ :

$$
\begin{aligned}
Z_{2}^{(4)}= & 0.20502387152777 \ldots \varepsilon^{-4}+0.59774667245370 \ldots \varepsilon^{-3}-0.89328249574801 \ldots \varepsilon^{-2} \\
& -6.18821133900575 \ldots \varepsilon^{-1}-17.2691387464077 \ldots+O(\varepsilon),
\end{aligned}
$$

which is in good agreement with the previous numerical value of Ref. [14]

$$
Z_{2}^{(4)}=0.20500(37) \varepsilon^{-4}+0.5980(27) \varepsilon^{-3}-0.895(21) \varepsilon^{-2}-6.18(17) \varepsilon^{-1}-17.4(1.6)+O(\varepsilon) .
$$

\section{Conclusions}

- fitting analytically families of master integrals is complicated;

- it needs very high precision numerical values (up to 9600 digits);

- it needs a deep analysis of some key master integrals;

- in the case of $g-2$ only a few percent of the total number of elements of the basis with polylogarithms of complex argument are needed;

- the current elliptical basis suffices to fit $F_{2}(0)^{(4)}, Z_{2}^{(4)}$ and $Z_{m}^{(4)}$;

- some guide to promptly identify only the needed elements of the basis would be useful, especially at 5-loop level.

\section{References}

[1] S. Laporta, Phys. Lett. B 772 (2017) 232.

[2] K. G. Chetyrkin and F. V. Tkachov, Nucl. Phys. B 192 (1981) 159.

[3] F. V. Tkachov, Phys. Lett. 100B (1981) 65.

[4] S. Laporta, Int. J. Mod. Phys. A 15 (2000) 5087.

[5] S. Laporta, Phys. Lett. B 504 (2001) 188.

[6] H. R. P. Ferguson and D. H. Bailey, RNR Technical Report RNR-91-032.

[7] D. H. Bailey and D. J. Broadhurst, Math. Comput. 70 (2001) 1719.

[8] E. Remiddi and J. A. M. Vermaseren, Int. J. Mod. Phys. A15 (2000) 725.

[9] Multiprecision fortran code from David Bailey, implementing sequential and parallel PSLQ http://crd-legacy.lbl.gov/dhbailey/mpdist/

[10] M. J. Levine and R. Roskies, Phys. Rev. D 9 (1974) 421.

[11] S. Laporta, Int. J. Mod. Phys. A 23 (2008) 5007.

[12] Y. Zhou, arXiv:1801.02182 [math.CA].

[13] S. Laporta and E. Remiddi, Nucl. Phys. B 704 (2005) 349.

[14] P. Marquard, A. V. Smirnov, V. A. Smirnov and M. Steinhauser, Phys. Rev. D 97, no. 5, 054032 (2018) 